Rev. Téc. Ing. Univ. Zulia. Vol. 44, No. 2, Mayo-Agosto, 2021, 83-91

\title{
Evaluación del comportamiento del acero 1,25Cr0,5Mo en condiciones de fluencia lenta
}

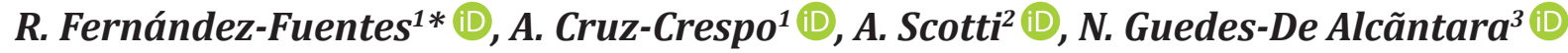

${ }^{1}$ Centro de Investigaciones de Soldadura, Facultad de Ingeniería Mecánica e Industrial, Universidad Central

"Marta Abreu" de Las Villas, CP 54 830, Santa Clara, Cuba.

${ }^{2}$ Universidade Federal de Uberlândia - UFU, Uberlândia, Brasil.

${ }^{3}$ Universidade Federal de São Carlos - UFSCar, São Carlos, Brasil.

*Autor de correspondencia: rfernandez@uclv.edu.cu

https://doi.org/10.22209/rt.v44n2a03

Recepción: 05 de agosto 2020 | Aceptación: 31 de enero 2021| Publicación: 15 de abril de 2021

\section{Resumen}

Los estudios sobre la influencia del tratamiento térmico pos soldadura en la microestructura y comportamiento mecánico de tuberías de vapor, se han enfocado en la región de la soldadura (metal fundido y zona afectada por el calor); sin embargo, dicho proceso tiene un efecto térmico que va más allá de esta región, el cual no ha sido suficientemente detallado. El presente trabajo tuvo como objetivo evaluar el efecto del tratamiento térmico sobre la microsetructura y el comportamiento en fluencia lenta de la región que no es térmicamente afectada por la soldadura. El material analizado fue tomado de una tubería de vapor (acero 1,25Cr0,5Mo, 20 años envejecido en servicio a $480{ }^{\circ} \mathrm{C}$ ). Una parte de la tubería fue sometida a tratamiento térmico, con parámetros de acuerdo con el código ASME de calderas y recipientes a presión. Se obtuvo el tiempo de rotura en ensayo de fluencia lenta y la microestructura del material envejecido en servicio, así como del tratado térmicamente. El tratamiento térmico del acero 1,25Cr0,5Mo envejecido en servicio aumentó el tamaño de los precipitados presentes en el interior de la ferrita y disminuyó la resistencia a la fluencia lenta.

Palabras claves: Acero 1,25Cr0,5Mo, tratamiento térmico pos soldadura, resistencia a la fluencia lenta.

\section{Evaluation of 1.25Cr0.5Mo steel behaviour in creep conditions}

\begin{abstract}
Studies about the influence of the post weld heat treatment on microstructures and mechanical behaviors of steam pipelines have focused on the weld region (fusion and heat-affected zones). However, this process has a thermal effect that surpasses this region, which has not been sufficiently detailed. The aim of the present work consisted in evaluating the effect of the heat treatment on the microstructure and creep behavior in the region that is not affected by welding thermal cycle. The analyzed material was taken from a main steam pipe (1.25Cr0.5Mo steel, 20 years in service aged at $480{ }^{\circ} \mathrm{C}$ ). A piece of the pipeline was subjected to heat treatment, with parameters according to the ASME boiler and pressure vessel code. The creep rupture time and the microstructure were obtained from the in service aged material, as well as from the heat-treated one. The heat treatment of in service aged $1.25 \mathrm{Cr} 0.5 \mathrm{Mo}$ steel increased the amount of the inside ferrite grain precipitates and reduced the creep resistance.
\end{abstract}

Key words: $1.25 \mathrm{Cr} 0.5 \mathrm{Mo}$ steel, posweld heat treatment, creep resistance. 


\section{Introducción}

El fenómeno de fluencia lenta se pone de manifiesto en un grupo de componentes industriales sometidos a condiciones de trabajo, que combinan la acción de carga mecánica y temperatura; como ejemplo se pueden mencionar, los generadores de vapor y las tuberías destinadas a su conducción. Para garantizar una explotación segura y duradera de dichos componentes bajo las mencionadas condiciones de trabajo, se han desarrollado, entre otros, los aceros ferríticos de baja aleación al Cr-Mo (Robertson, 2014). En estos aceros la resistencia a la fluencia lenta se logra, básicamente, por la acción de los mecanismos de endurecimiento por solución sólida y precipitación (Muránsky et al., 2020).

En el diseño de los referidos componentes, para cada marca de acero se adopta una tensión admisible que garantice un trabajo seguro durante al menos $10^{5} \mathrm{~h}$ a la temperatura de operación. Sin embargo, los factores de seguridad aplicados permiten que en la práctica, los componentes fabricados con aceros ferríticos al Cr-Mo puedan alcanzar y sobrepasar los $2 \cdot 10^{5} \mathrm{~h}$ explotación (Victoria y Félix, 2007). No obstante, se dan casos en los que surgen fallas prematuras, incluso antes de las $10^{5} \mathrm{~h}$, asociadas a diferentes fenómenos de degradación (Furtado y Le-May, 2004; Victoria y Félix, 2007).

Cuando dichas fallas ocurren, en casos técnico-económicamente justificados, se procede a la reparación, retirando la zona dañada y soldando un inserto de material nuevo. Los códigos que se aplican para este tipo de reparación, entre los cuales se encuentran los códigos ASME (ASME, 2019) y JIS B8267 (JSA, 2015), contemplan los requerimientos tecnológicos que garantizan la calidad de la unión soldada apenas en materiales nuevos. Lo que significa que el procedimiento de reparación se torne complejo cuando involucra al material envejecido en servicio, porque en la mayoría de los casos, se desconoce su estado microestructural y comportamiento mecánico. Tal situación impone la necesidad de conocer el comportamiento del material sometido a fluencia lenta por tiempos prolongados.

Este problema se complica en aquellos casos en los que se requiere la aplicación de tratamiento térmico posterior a la soldadura, notándose discrepancias en la literatura (Parker y Stratford, 1995), en relación a si el mismo es favorable o no desde el punto de vista de la vida residual. En tal sentido, se observa que el enfoque fundamental de los correspondientes estudios ha estado centrado en el efecto del tratamiento térmico sobre la zona afectada térmicamente (ZAT), a pesar de que el mismo también ejerce influencia térmica de consideración sobre una región que se extiende más allá de la ZAT.

Con base en lo planteado anteriormente, el presente trabajo tuvo como objetivo, evaluar el comportamiento de la microestructura y de la resistencia a la fluencia lenta del acero $1,25 \mathrm{Cr} 0,5 \mathrm{Mo}$, envejecido en servicio y sometido a tratamiento térmico similar al que se aplica pos soldadura.

\section{Materiales y Métodos}

Para realizar el estudio se empleó el material de una sección retirada de una tubería de la línea de salida de un generador de vapor, que estuvo en el entorno de 20 años en servicio, a la temperatura de $480{ }^{\circ} \mathrm{C}$. La composición química del material de la tubería (Tabla 1), determinada mediante espectrometría de absorción atómica, se corresponde con la del acero tipo 1,25Cr0,5Mo; según la norma ASTM A387 (ASTM, 2011a).

Tabla 1. Composición química elemental del material de la tubería (\% en masa).

\begin{tabular}{ccccccccccc}
\hline $\mathbf{C}$ & $\mathbf{S i}$ & $\mathbf{M n}$ & $\mathbf{P}$ & $\mathbf{S}$ & $\mathbf{C r}$ & $\mathbf{M o}$ & $\mathbf{W}$ & $\mathbf{T i}$ & $\mathbf{V}$ & $\mathbf{F e}$ \\
\hline 0,12 & 0,38 & 0,41 & $<0,03$ & $<0,03$ & 1,24 & 0,49 & $<0,01$ & $<0,01$ & $<0,01$ & base \\
\hline
\end{tabular}

Una parte de la referida sección fue sometida a tratamiento térmico en horno mufla, con un régimen similar al recomendado por el código ASME (ASME, 2019) para el tratamiento térmico pos soldadura: velocidad de calentamiento de $200{ }^{\circ} \mathrm{C} / \mathrm{h}$ y permanencia de $1,5 \mathrm{~h}$ a la temperatura de $700^{\circ} \mathrm{C}$. De esta manera, se evaluaron dos condiciones del material:

a. Material envejecido en servicio (MES). Se asocia al material directamente retirado de la tubería.

b. Material envejecido en servicio y sometido a tratamiento térmico (MES+TT).

A partir de ambas condiciones, se aplicaron ensayos de fluencia lenta a carga constante, en probetas cilíndricas (diámetro $6 \mathrm{~mm}$ y longitud útil $36 \mathrm{~mm}$ ), de acuerdo con la norma ASTM E-139 (ASTM, 2011d). Cada condición ensayada fue resultado de la combinación de valores de la tensión y de la temperatura, de acuerdo con la Tabla 2. 
Tabla 2. Combinación de valores de la tensión y de la temperatura para cada condición del material (MES y MES+TT).

\begin{tabular}{cccc}
\hline \multirow{2}{*}{ Temperatura $\left({ }^{\circ} \mathbf{C}\right)$} & \multicolumn{3}{c}{ Tensión (MPa) } \\
\cline { 2 - 4 } & $\mathbf{1 0 0}$ & $\mathbf{1 2 5}$ & $\mathbf{1 5 0}$ \\
\hline \multirow{2}{*}{550} & MES & MES & MES \\
& MES+TT & & MES+TT \\
575 & MES & MES & MES \\
& MES+TT & & MES+TT \\
\multirow{2}{*}{600} & MES & MES & MES \\
& MES+TT & & MES+TT \\
\hline
\end{tabular}

MES: material envejecido en servicio, MES+TT: material envejecido en servicio y sometido a tratamiento térmico.

A partir de los resultados del ensayo de fluencia lenta, se evaluó:

a. El efecto del envejecimiento en servicio sobre el tiempo de rotura en fluencia lenta, para lo cual se compararon los resultados de la condición MES, con valores reportados por Demirkol (1999), para el mismo tipo de acero en estado de entrega con composición química y microestructura compatibles con las del material estudiado.

b. La influencia del tratamiento térmico sobre el tiempo de rotura en fluencia lenta, para lo cual se comparó el comportamiento de las condiciones MES y MES+TT (para combinaciones similares de tensión y temperatura).

La preparación de la superficie de las probetas para la adquisición de las imágenes de la microestructura, de acuerdo con las recomendaciones de la norma ASTM E-3 (ASTM, 2011b), consistió en el desbaste con papel esmeril entre 120 y 1000, seguido del pulido con alúmina de 3 y $1 \mu \mathrm{m}$, aplicándose NITAL al $1 \%$ como reactivo para el ataque químico por inmersión durante $10 \mathrm{seg}$, de acuerdo con la norma ASTM E-407 (ASTM, 2011c).

La observación de la microestructura se realizó en un microscopio electrónico de barrido (Zeiss EVO MA10), con adquisición de imágenes digitales de 3072 x 2304 píxeles y resolución de $10 \mathrm{~nm} /$ píxel. Mediante el uso del programa de computación Image J, se realizó el procesamiento digital de imagen, y de manera automatizada fue determinado el diámetro equivalente de los precipitados, en función del área ocupada por cada sección de los mismos en la imagen.

Se efectuó un análisis comparativo de la distribución de tamaños de precipitados intra-granulares de las condiciones estudiadas, de modo que, a partir de los tamaños de partículas, se confeccionaron los histogramas de frecuencias relativas (empleando en todos los casos 15 clases de tamaño igual a $40 \mathrm{~nm}$ ) y se determinó la densidad de partículas en cada intervalo de clase (Smith y Jordan, 1964).

Los valores experimentales de la densidad de partículas fueron ajustados a un modelo de densidad de probabilidad tipo log-normal (ecuación 1; Endo, 2009) y se obtuvieron los parámetros de la función (media geométrica y desviación estándar). Para establecer la calidad del ajuste se empleó el valor del coeficiente de determinación y la "prueba F" en el análisis de varianza (Krishnamoorthy y Mathew, 2003; Sultan y Ahmad, 2013; Martínez et al., 2015).

$$
f\left(D_{i}\right)=\frac{1}{\sqrt{2 \pi} D\left(\ln \sigma_{g}\right)} \exp \left[-\frac{1}{2}\left(\frac{\ln \left(D_{i} / D_{g}\right)}{\ln \sigma_{g}}\right)^{2}\right]
$$

Donde: $\mathrm{D}_{\mathrm{i}}$ : diámetro $(\mathrm{mm}), \mathrm{D}_{\mathrm{g}}$ : media geométrica del diámetro $(\mathrm{mm}), \boldsymbol{\sigma}_{\mathrm{g}}$ : desviación geométrica estándar.

\section{Resultados y Discusión}

En la condición MES se apreció una microestructura predominantemente ferrítica, acompañada de perlita laminar (Figura 1a); en la ferrita se observaron precipitados finos intragranulares e intergranulares (Figura 1b). El acero $1,25 \mathrm{Cr} 0,5 \mathrm{Mo}$ cualitativamente presenta este tipo de microestructura en estado de entrega, cuando es sometido a un recocido, mientras que, para el caso de un normalizado con revenido, en lugar de perlita, se forma bainita (Viswanathan, 1995). Una precipitación similar (intragranular e intergranular) fue observada en el acero $2,25 \mathrm{Cr} 1 \mathrm{Mo}$, tanto en estado de 
entrega como expuesto en servicio por un largo período (Yang, 1993). La formación de este tipo de precipitación también ha sido observada en muestras de esta familia de aceros, sometidos al ensayo de fluencia lenta (Afrouz et al., 1983).
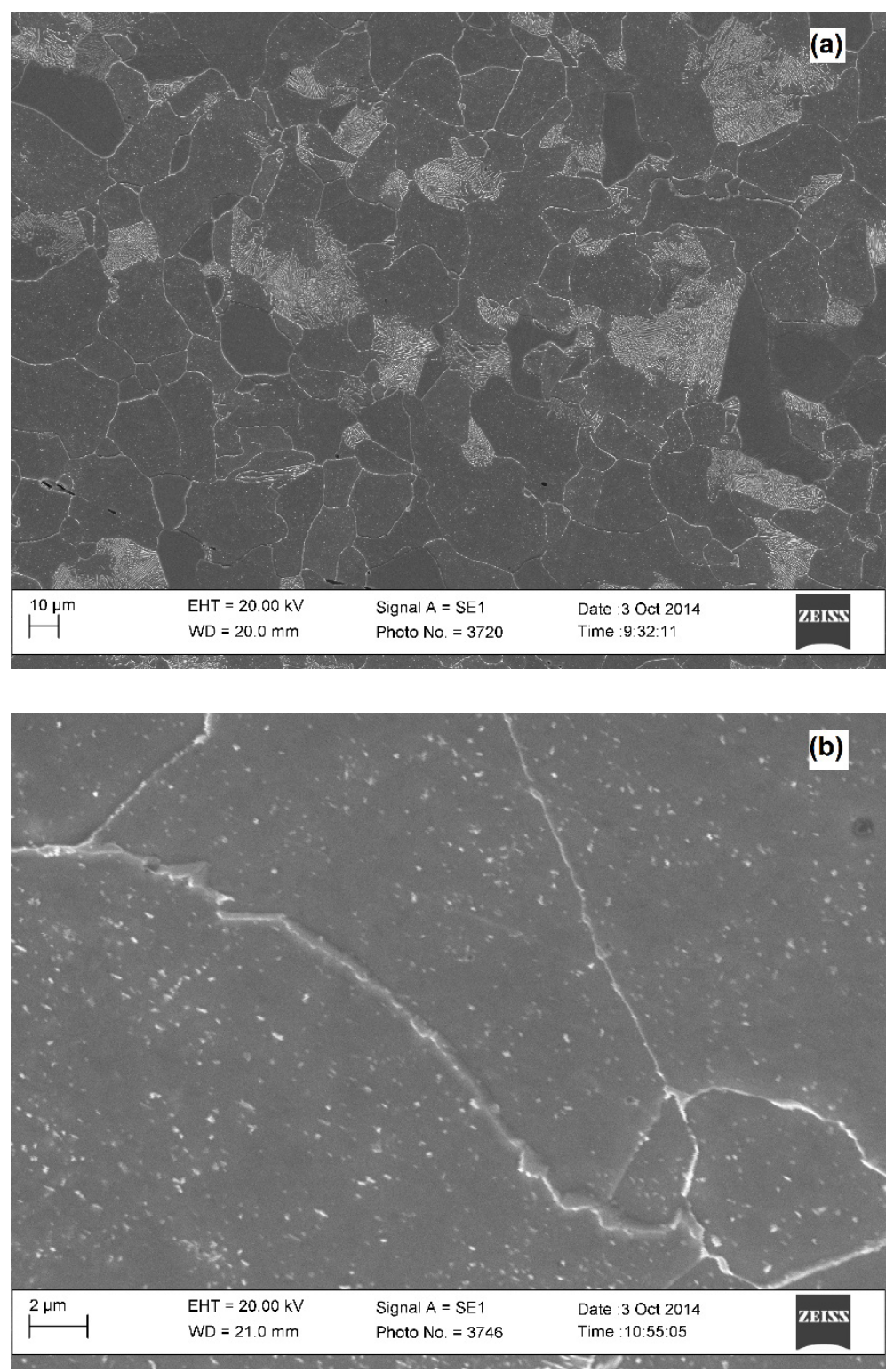

Figura 1. (a) Microestructura ferrítico-perlítica de la condición de material envejecido en servicio (MES), (b) Precipitación en el interior y en los contornos de grano de la ferrita en la condición de material envejecido en servicio (MES).

El referido tipo de microestructura se conserva en la condición del MES+TT, aunque, en esta condición, se apreciaron precipitados de mayor tamaño en el interior de la ferrita (Figura 2), en comparación con los observados en la condición MES (Figura 1b). Esta diferencia se evidencia cuantitativamente en los histogramas de frecuencia relativa de tamaños de precipitados y las curvas de densidad de partículas (Figuras 3 y 4 , respectivamente). 


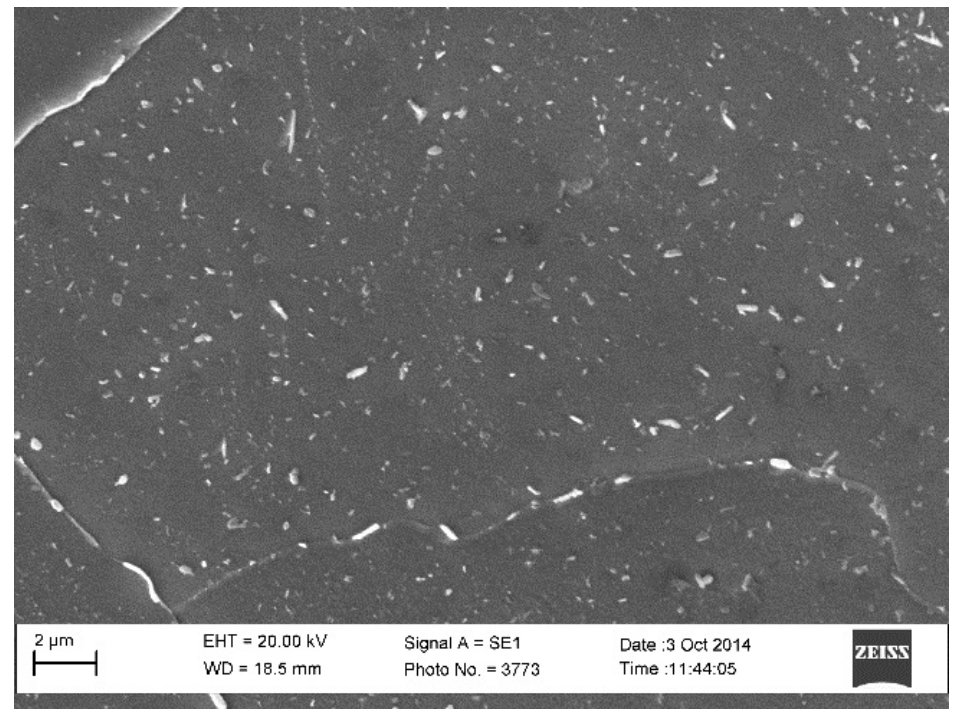

Figura 2. Microestructura de la condición de material envejecido en servicio con tratamiento térmico (MES+TT).

De acuerdo con la Figura 3, con el tratamiento térmico (condición MES+TT) disminuyó considerablemente la frecuencia relativa de la clase $40-80 \mathrm{~nm}$, mientras que se incrementa respectivamente en el resto de las clases (para tamaños por encima de $80 \mathrm{~nm}$ ). Con relación a la densidad de partículas, para ambas condiciones se obtuvo un adecuado ajuste al modelo tipo lognormal (coeficiente de determinación de 0,998 y probabilidad $p<0,001$ para la condición MES, y de 0,999 con $p<0,001$ para la condición MES+TT). En este caso se apreció que el tratamiento térmico modificó la curva de densidad de probabilidad ajustada (Figura 4), trayendo como resultado un incremento de la moda (de $88 \mathrm{~nm}$ para la condición MES a $105 \mathrm{~nm}$ para la condición MES+TT) y de la media geométrica (de 101 a $117 \mathrm{~nm}$, respectivamente).

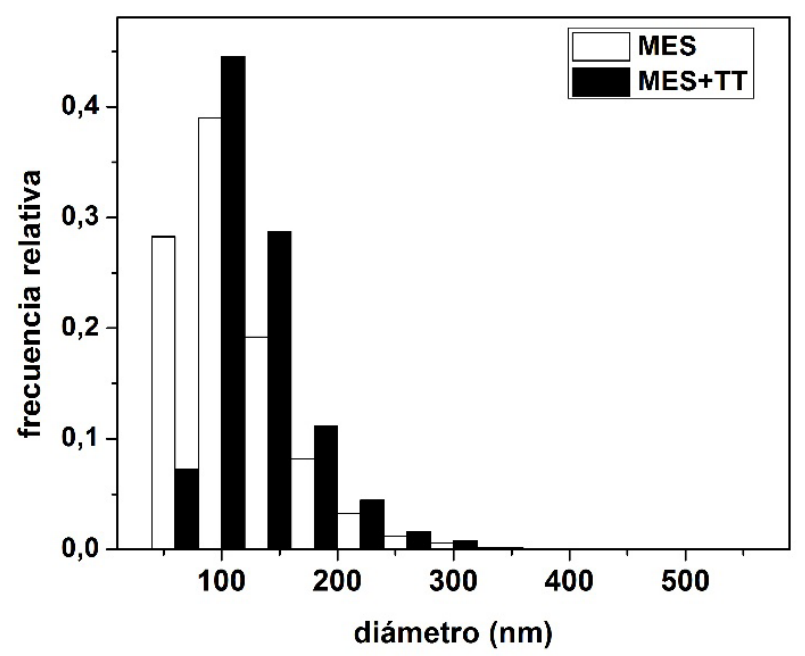

Figura 3. Histogramas de frecuencia relativa de tamaños de precipitados de las condiciones MES (material envejecido en servicio) y MES+TT (material envejecido en servicio y sometido a tratamiento térmico). 


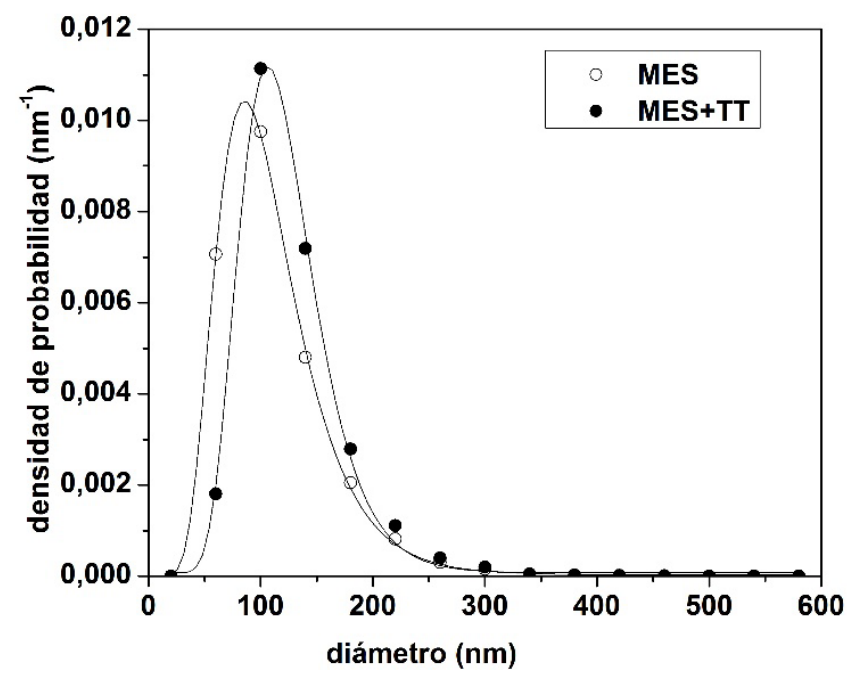

Figura 4. Puntos experimentales y curvas de densidad de partículas de las condiciones MES (material envejecido en servicio) y MES+TT (material envejecido en servicio y sometido a trtatamiento térmico).

El efecto que sobre la distribución del tamaño de precipitados provocó el tratamiento térmico pos soldadura, se asocia con el hecho de que los precipitados estables de mayor tamaño crecen a expensas de la disolución de los más pequeños, como resultado del proceso denominado crecimiento competitivo (Ouden, 2015), reportado para aceros de baja aleación al CrMo (Gustafson y Hattestrand, 2002), cuya fuerza motríz es la disminución de la energía libre del sistema provocada por el descenso de la energía superficial.

La comparación gráfica de los resultados del ensayo de fluencia lenta para las condiciones MES y MES+TT (Figura 5), indica que, para las mismas combinaciones experimentales de tensión y temperatura, la condición MES+TT presentó un menor tiempo de rotura con relación a la condición MES. La reducción del tiempo de rotura observada significa un efecto desfavorable del tratamiento térmico sobre la resistencia a la fluencia lenta, lo cual coincide con reportes de la literatura para esta familia de aceros (Arnswald et al., 1986), a pesar de que también hay reportes sobre un efecto favorable (Lundin y Wang 1989).

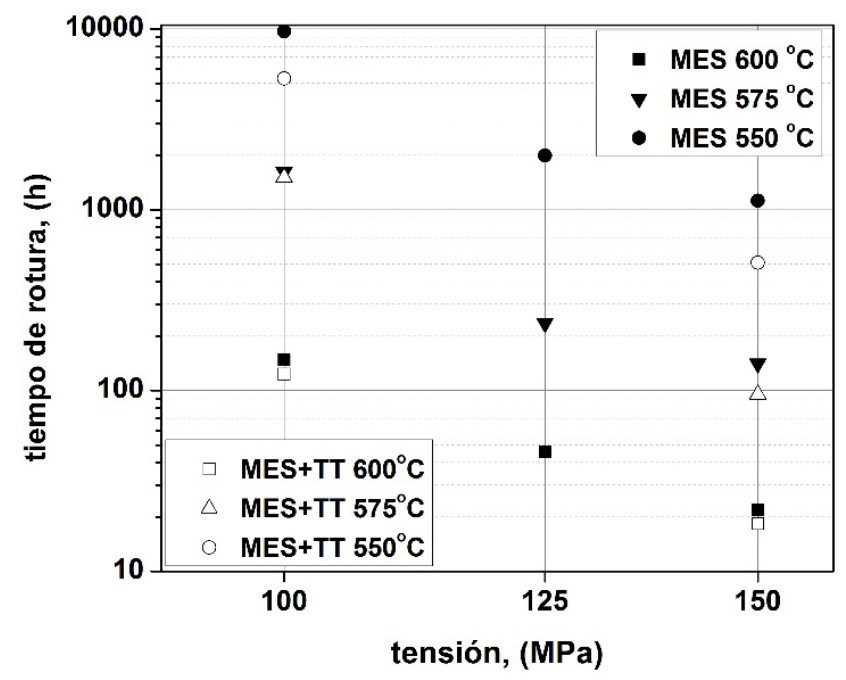

Figura 5. Tiempo de rotura por fluencia lenta de las condiciones MES (material envejecido en servicio) y MES+TT (material envejecido en servicio y sometido a tratamiento térmico), para diferentes combinaciones de tensión y temperatura de ensayo. 
Para iguales combinaciones de tensión (100 y $150 \mathrm{MPa}$ ) y temperatura $\left(550\right.$ y $\left.600{ }^{\circ} \mathrm{C}\right)$, en la Figura 6 se muestran los valores experimentales del tiempo de rotura de las condiciones MES y del MES+TT, así como las líneas de tendencia reportadas por Dermikol (1999) para el acero 1,25Cr0,5Mo en estado de entrega con composición química y microestructura similares a las determindas en las muestras bajo estudio en el presente. En este caso, la comparación gráfica muestra que tanto la condición MES como MES+TT, presentaron menor tiempo de rotura en comparación con el material en estado de entrega, y por ende menor resistencia a la fluencia lenta.

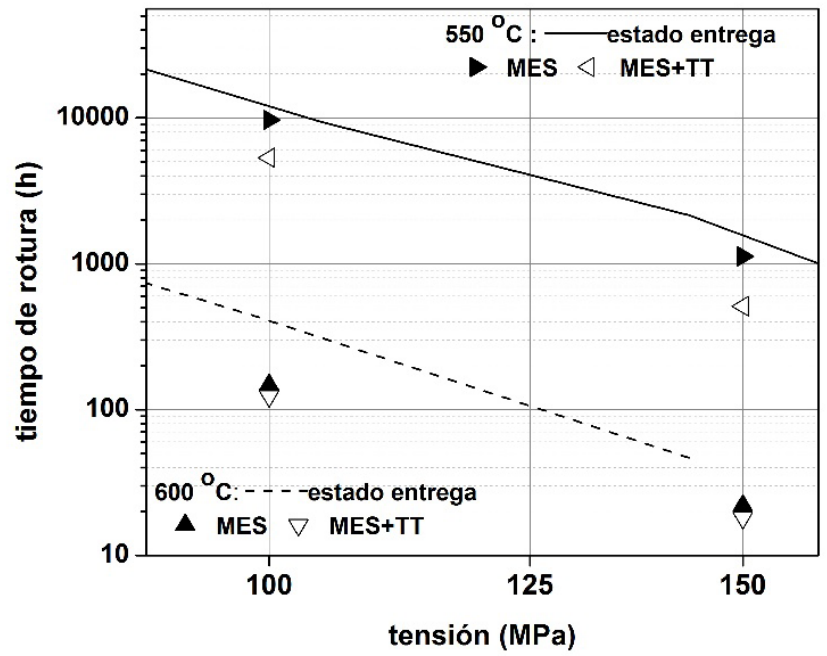

Figura 6. Tiempo de rotura por fluencia lenta de las condiciones experimentales MES (material envejecido en servicio) y MES+TT (material envejecido en servicio y sometido a tratamiento térmico) y del material en estado de entrega

(Demirkol, 1999).

La evaluación cuantitativa de la reducción del tiempo de rotura en las diferentes condiciones se efectuó sobre la base de modelos estadísticos (ecuaciones 2, 3 y 4, para las condiciones MES, MES+TT y estado de entrega, respectivamente) obtenidos mediante el análisis de regresión múltiple. Para este análisis, los valores experimentales se ajustaron a diferentes modelos recomendados en la literatura para tales fines (Manson y Ensign, 1979). Para cada condición se adoptó aquel modelo en el que el análisis de varianza mostró valores estadísticamente significativos de las correspondientes pruebas $\left(p<0,05\right.$ para las pruebas $\mathrm{F}$ y $\mathrm{t}$, respectivamente) y mayor coeficiente de determinación múltiple ajustado ( $\mathrm{R}^{2}$ ajustado).

$$
\begin{aligned}
& \log t_{r}=13,96-19,3 \cdot \log (S)+1,18 \cdot 10^{4}(\log (S) / T) \\
& \log t_{r}=13,0-17,5 \cdot \log (S)+1,06 \cdot 10^{4}(\log (S) / T) \\
& \log t_{r}=8,5-15,9 \cdot \log (S)+1,2 \cdot 10^{4}(\log (S) / T)-13,1(S / T)
\end{aligned}
$$

Donde: $t_{\mathrm{r}}$ : tiempo de rotura $(\mathrm{h}), \mathrm{S}$ : tensión $(\mathrm{MPa})$, T: temperatura $(\mathrm{K})$.

De acuerdo con los modelos ajustados, para la tensión admisible del acero 1,25Cr0,5Mo a la temperatura de operación (94 MPa a 480 oC, según el código ASME; ASME, 2019), se obtuvo que el tiempo de rotura de la condición MES+TT representa un 32,2 \% del correspondiente a la condición MES, mientras que el tiempo de rotura de la condición MES representa un $65 \%$ del correspondiente al material en estado de entrega, y el tiempo de rotura de la condición MES+TT representa un 20,4 \% del correspondiente al material en estado de entrega. De esta manera, se pone de manifiesto el efecto perjudicial del tratamiento térmico pos soldadura sobre la resistencia a la fluencia lenta del acero $1,25 \mathrm{Cr} 0,5 \mathrm{Mo}$ envejecido en servicio, expresado en una reducción del tiempo de rotura de un 67,8 \%. Este comportamiento mecánico se corresponde con el comportamiento microestructural, que muestra que el tratamiento térmico provocó una disminución de la frecuencia relativa de precipitados pequeños, con aumento de la de los mayores. Ello trae como consecuencia un aumento de la distancia entre partículas, con detrimento del efecto del incremento de la resistencia mecánica mediante la precipitación (Abbaschian et al., 2009).

\section{Conclusiones}

El acero 1,25Cr0,5Mo con 20 años de operación en una tubería de vapor, conserva cualitativamente la 
microestructura del tipo ferrítico-perlítica con precipitados en el interior y los contornos de grano de ferrita, la cual es típica del estado de entrega normalizado.

El tratamiento térmico con régimen similar al requerido pos soldadura, no modifica este tipo de microestructura; sin embargo, como consecuencia del fenómeno de crecimiento competitivo, el mismo trae como resultado cambios cuantitativos de la distribución de tamaño de los precipitados presentes en el interior de la ferrita, expresados por una disminución de la cantidad de partículas menores que $80 \mathrm{~nm}$ y aumento de la cantidad de partículas mayores.

En el acero 1,25Cr0,5Mo, tanto envejecido en servicio como sometido a tratamiento térmico con régimen similar al requerido pos soldadura, la densidad de partículas en el interior de la ferrita obedece a una función de distribución tipo lognormal, manifestándose un incremento de $16 \%$ de la media geométrica del diámetro equivalente por efecto del tratamiento térmico.

El tiempo de rotura en fluencia lenta se ajusta adecuadamente a un modelo del tipo $\log t_{r}=A+B \cdot \log (S)+C(\log (S) / T)+D(S / T)$, donde: A, B, C y D son los parámetros del modelo, t el tiempo de rotura, h, $\mathrm{S}$ la tensión, MPa y T la temperatura ${ }^{\circ} \mathrm{C}, \mathrm{K}$.

El tratamiento térmico del acero 1,25Cr0,5Mo previamente envejecido en servicio, con parámetros similares al requerido pos soldadura, reduce en un $67,8 \%$ el tiempo de rotura en fluencia lenta, lo cual se asocia con el correspondiente incremento del tamaño de los precipitados en el interior de la ferrita, conducente al detrimento del efecto de incremento de la resistencia mecánica por precipitación.

\section{Agradecimientos}

Este trabajo fue posible gracias al apoyo brindado por LAPROSOLDA y LDTAD de UFU, DEMa y CCDM de UFSCar y por el convenio CAPES-Brasil/MES-Cuba.

\section{Referencias Bibliográficas}

Abbaschian, R., Abbaschian, L., Reed-Hill, R. (2009). Physical Metallurgy Principles, USA: Cengage Learning.

Afrouz, A., Collins, J., Pilkington, R. (1983). Microestructural examination of 1Cr0,5Mo steel during creep. Metals Technology, 10(12), 461-463.

ASTM. (2011a). ASTM A387/A387M-1: Standard specification for pressure vessel plates, alloy steel, chromium-molybdenum. American Society for Testing and Materials: West Conshohocken.

ASTM. (2011b). ASTM E3-11: Standard guide for preparation of metallographic specimens. American Society for Testing and Materials: West Conshohocken.

ASTM. (2011c). ASTM E407-11: Standard practice for microetching metals and alloys. American Society for Testing and Materials: West Conshohocken.

ASTM. (2011d). ASTM E-139: Standard practice for creep, creep-rupture, and stress-rupture tests of metallic materials. American Society for Testing and Materials: West Conshohocken.

ASME. (2019). Rules for construction of pressure vessels. Boiler and pressure vessel code. American Society of Mechanical Engineers: West Conshohocken.

Arnswald, W., Blum, R., Neubauer, B., Poulsen, K. (1986). Remaining life affected by repair welds, In: International Conference on Creep, JSME/IMechE/ASME/ASTM: Tokyo.

Demirkol, M. (1999). On the creep strength-rupture ductility behaviour of 1.25Cr-0.5Mo low alloy steel. Journal of Engineering and Environmental Science, (23), 389-401.

Endo, Y. (2009). Estimate of confidence intervals for geometric mean diameter and geometric standar deviation of lognormal size distribution. Power Technology, 193(2), 154-161.

Furtado, H., Le-May, I. (2004). High temperature degradation in power plants and refineries. Materials research, 7(1), 103110.

Gustafson, A., Hattestrand, M. (2002). Coarsening of precipitates in an advanced creep resistant 9\% chromium steelquantitative microscopy and simulations. Materials Science and Engineering, 333(1), 279-286.

JSA. (2015). Construction of pressure vessel. JIS B8267. Japanese Standards Association: Tokyo. 
Krishnamoorthy, K., Mathew, T. (2003). Inferences on the means of lognormal distributions using generalized p-values and generalized confidence intervals. Journal of Statistical Planning and Inference, 115, 103-121.

Lundin , C., Wang, Y. (1989). Repair welding of service exposed Cr-Mo steel weldments. Welding Research Council, $384,1-39$.

Manson, S., Ensign, C. (1979). A quarter century of progress in the development of correlation and extrapolation methos for creep rupture data. Journal of Engineering and Materials Technology, 101(10), 317-325.

Martínez, L., Linares, J., Martínez, R., Oliva, H. (2015). Regression models for prediction of properties of pvc compounds considering the effects of additives dosis. Revista Técnica de la Facultad de Ingeniería. Universidad de Zulia, 38(3), $266-274$.

Muránsky, O., Zhu, H., Lim, L., Short, K., Cairney, J., Drew, M. (2020). On the evolution of mechanical properties and microstructure of ferritic-bainitic (FB) 2.25Cr-1Mo (Grade 22) steel during high-temperature creep. Materialia, (9), 1-14.

Ouden, D. (2015). Mathematical Modelling of nucleating and growing precipitates: distributions and interfaces. Delft University of Technology, Netherlands.

Parker, J. and Stratford, G. (1995). Effect of heat treatment on creep and fracture behavior of 1,25Cr0,5Mo steel. Materials Science and Technology, 11(12), 1267-1273.

Robertson, D. (2014). Traditional low alloy steels in power plantdesig, development and applications. In: Coal Power Plant Materials and Life Assessment. Ed. Shibli, A. Amsterdam, The Netherlands: Woodhead Publishing.

Smith, J., Jordan, M. (1964). Mathematical and graphical interpretation of the log-normal law for particle size distribution analysis. Journal of Colloid Science, 19(6), 549-559.

Sultan, R., Ahmad, S. (2013). Comparison of parameters of lognormal distribution based on the classical and posterior estimates. Journal of Modern Applied Statistical, 12(2), 304-313.

Victoria, H., Félix, S. (2007). Tendencias actuales para determinar la degradación de los materiales metálicos de componentes industriales. Nucleus, (42), 17-28.

Viswanathan, R. (1993). Damage mechanisms and life assessment of high-temperature components. Ohio: Carnes Publication Services.

Yang, R. (1993). Microestructural examination of 2.25Cr1Mo steel pipes after extended service. Materials Characterization, 30(2), 75-88. 


\section{REVISTA TECNICA}

DE LA FACULTAD DE INGENIERIA

UNIVERSIDAD DEL ZULIA

Vol. 44. №2, Mayo - Agosto, 2021

Esta revista fue editada en formato digital y publicada en Abril de 2021, por el Fondo Editorial Serbiluz, Universidad del Zulia. Maracaibo-Venezuela

www.luz.edu.ve

www.serbi.luz.edu.ve

www.produccioncientificaluz.org 